\title{
The Arithmetical Complexity of Dimension and Randomness*
}

\author{
John M. Hitchcock ${ }^{\dagger} \quad$ Jack H. Lutz ${ }^{\ddagger} \quad$ Sebastiaan A. Terwijn ${ }^{\S}$
}

\begin{abstract}
Constructive dimension and constructive strong dimension are effectivizations of the Hausdorff and packing dimensions, respectively. Each infinite binary sequence $A$ is assigned a dimension $\operatorname{dim}(A) \in[0,1]$ and a strong dimension $\operatorname{Dim}(A) \in[0,1]$.

Let $\mathrm{DIM}^{\alpha}$ and $\mathrm{DIM}_{\mathrm{str}}^{\alpha}$ be the classes of all sequences of dimension $\alpha$ and of strong dimension $\alpha$, respectively. We show that $\mathrm{DIM}^{0}$ is properly $\Pi_{2}^{0}$, and that for all $\Delta_{2}^{0}$-computable $\alpha \in(0,1]$, $\mathrm{DIM}^{\alpha}$ is properly $\Pi_{3}^{0}$.

To classify the strong dimension classes, we use a more powerful effective Borel hierarchy where a co-enumerable predicate is used rather than a enumerable predicate in the definition of the $\Sigma_{1}^{0}$ level. For all $\Delta_{2}^{0}$-computable $\alpha \in[0,1)$, we show that $\operatorname{DIM}_{\text {str }}^{\alpha}$ is properly in the $\Pi_{3}^{0}$ level of this hierarchy. We show that $\mathrm{DIM}_{\mathrm{str}}^{1}$ is properly in the $\Pi_{2}^{0}$ level of this hierarchy.

We also prove that the class of Schnorr random sequences and the class of computably random sequences are properly $\Pi_{3}^{0}$.
\end{abstract}

Keywords: arithmetical hierarchy, Wadge reductions, constructive dimension, Schnorr randomness, computable randomness

\section{Introduction}

Hausdorff dimension - the most extensively studied fractal dimension - has recently been effectivized at several levels of complexity, yielding applications to a variety of topics in theoretical computer science, including data compression, polynomial-time degrees, approximate optimization, feasible prediction, circuit-size complexity, Kolmogorov complexity, and randomness $[15,16,3,1,7,5,8,18]$. The most fundamental of these effectivizations is constructive dimension, which is closely related to Kolmogorov complexity and algorithmic randomness. Every subset $\mathcal{X}$ of $\mathbf{C}$, the Cantor space of all infinite binary sequences, is assigned a constructive dimension $\operatorname{cdim}(\mathcal{X}) \in[0,1]$. Informally, this dimension is determined by the maximum rate of growth that a lower semicomputable martingale can achieve on all sequences in $\mathcal{X}$.

Just as Martin-Löf [17] used constructive measure to define the randomness of individual sequences, Lutz [16] used constructive dimension to define the dimensions of individual sequences.

\footnotetext{
${ }^{*}$ A preliminary version of the paper appeared in Proceedings of the Annual Conference of the European Association for Computer Science Logic, Lecture Notes in Computer Science 2803, pages 241-254. Springer-Verlag, 2003.

${ }^{\dagger}$ Department of Computer Science, University of Wyoming, Laramie, WY 82071, USA. jhitchco@cs.uwyo.edu. This author's research was supported in part by National Science Foundation grant 9988483.

${ }^{\ddagger}$ Department of Computer Science, Iowa State University, Ames, IA 50011, USA. Iutz@cs.iastate.edu. This author's research was supported in part by National Science Foundation grants 9988483 and 0344187.

$\S$ Technische Universität Wien, Wiedner Hauptstrasse 8-10, A-1040 Vienna, Austria. terwijn@logic.at. Supported by the Austrian Research Fund (Lise Meitner grant M699-N05). Part of this author's research was done while visiting the second author at Caltech in January 2002.
} 
Each sequence $A \in \mathbf{C}$ is assigned a dimension $\operatorname{dim}(A) \in[0,1]$ by $\operatorname{dim}(A)=\operatorname{cdim}(\{A\})$. Every Martin-Löf random sequence has dimension 1 , but there are nonrandom sequences with dimension 1. For every real number $\alpha \in[0,1]$, there is a sequence with dimension $\alpha$.

It is useful to understand the arithmetical complexity of a class of sequences. For example, knowing that RAND, the class of Martin-Löf random sequences, is a $\Sigma_{2}^{0}$-class allows the application of Kreisel's Basis Lemma (which asserts that every infinite recursive tree has a $\Delta_{2}^{0}$ branch $[13,20]$ ) to give a short proof [28] that

$$
\operatorname{RAND} \cap \Delta_{2}^{0} \neq \emptyset
$$

For any $\alpha \in[0,1]$, let

$$
\operatorname{DIM}^{\alpha}=\{A \in \mathbf{C} \mid \operatorname{dim}(A)=\alpha\} .
$$

Lutz [16] showed that

$$
\operatorname{DIM}^{\alpha} \cap \Delta_{2}^{0} \neq \emptyset
$$

for any $\Delta_{2}^{0}$-computable $\alpha \in[0,1]$. As these dimension classes do not appear to be $\Sigma_{2}^{0}$, Lutz was unable to apply the Basis Lemma to them, so he used different techniques to prove (1.2).

We investigate the complexities of these dimension classes in terms of the arithmetical hierarchy of subsets of $\mathbf{C}$. We note that for the arithmetical hierarchy of sets of reals there is no natural completeness notion. We discuss various methods that can be used to classify sets in this hierarchy. We show that $\mathrm{DIM}^{0}$ is properly $\Pi_{2}^{0}$, and for all $\Delta_{2}^{0}$-computable $\alpha \in(0,1]$ we show that $\mathrm{DIM}^{\alpha}$ is properly $\Pi_{3}^{0}$. Therefore, the proof for (1.1) using Kreisel's Basis Lemma cannot be used directly to establish (1.2). (See however the comments made after Corollary 4.11.)

More recently, packing dimension, another important fractal dimension, has also been effectivized by Athreya, Hitchcock, Lutz, and Mayordomo [2]. At the constructive level, this is used in an analogous way to define the strong dimension $\operatorname{Dim}(A) \in[0,1]$ for every sequence $A$. For any $\alpha \in[0,1]$, let

$$
\operatorname{DIM}_{\text {str }}^{\alpha}=\{A \in \mathbf{C} \mid \operatorname{Dim}(A)=\alpha\} .
$$

To classify these strong dimension classes, we use a more powerful effective Borel hierarchy where a co-enumerable predicate is used rather than a enumerable predicate in the definition of the $\Sigma_{1}^{0}$ level. We show that $\operatorname{DIM}_{\text {str }}^{1}$ is properly in the $\Pi_{2}^{0}$ level of this stronger hierarchy. For all $\Delta_{2}^{0}-$ computable $\alpha \in[0,1)$, we show that $\mathrm{DIM}_{\text {str }}^{\alpha}$ is properly in the $\Pi_{3}^{0}$ level of this hierarchy. Apart from the dimension classes there are also other classes that are best classified in the extended Borel hierarchy. These include natural examples such as the computable sets (Theorem 4.4) and the 1-generic sets (Proposition 3.3).

Our techniques for classifying the dimension and strong dimension classes include Baire category, Wadge reductions, and Kolmogorov complexity. In Section 4.3 we point out that ad hoc methods are sometimes necessary.

Section 2 gives an overview of the randomness and dimension notions used in this paper. In Section 3 we introduce the stronger effective Borel hierarchy that we use for the strong dimension classes. Section 4 presents the classification of $\mathrm{DIM}^{\alpha}$ and $\mathrm{DIM}_{\mathrm{str}}^{\alpha}$.

We conclude the paper with Section 5 on effective randomness classes. We restate a result of Schnorr [22] concerning computable null sets of exponential order in terms of computable dimension and point out a relationship with Church randomness. We prove that the class of Schnorr random sequences and that the class of computably random sequences are properly $\Pi_{3}^{0}$. 


\section{Background on Randomness and Dimension}

This section provides an overview of the notions of randomness and dimension used in this paper. We write $\{0,1\}^{*}$ for the set of all finite binary strings and $\mathbf{C}$ for the Cantor space of all infinite binary sequences. In the standard way, a sequence $A \in \mathbf{C}$ can be identified with the subset of $\{0,1\}^{*}$ or $\mathbb{N}$ for which it is the characteristic sequence, or with a real number in the unit interval. The length of a string $w \in\{0,1\}^{*}$ is $|w|$. The string consisting of the first $n$ bits of $x \in\{0,1\}^{*} \cup \mathbf{C}$ is denoted by $x\lceil n$. We write $w \sqsubseteq x$ if $w$ is a prefix of $A$.

\subsection{Martin-Löf Randomness}

Martin-Löf [17] introduced the notion of a constructive null set. A set is constructively null if it can be covered by a uniform sequence of c.e. open sets that are shrinking in size. That is, $\mathcal{A} \subseteq \mathbf{C}$ is constructive null if $\mathcal{A} \subseteq \bigcap_{i} \mathcal{U}_{i}$, where $\left\{\mathcal{U}_{i}\right\}_{i \in \mathbb{N}}$ is uniformly c.e. such that $\mu\left(\mathcal{U}_{i}\right) \leq 2^{-i}$. The sequence $\left\{\mathcal{U}_{i}\right\}_{i \in \mathbb{N}}$ is called a Martin-Löf test. An individual sequence $A \in \mathbf{C}$ is Martin-Löf random if $\{A\}$ is not constructively null. The Martin-Löf random sequences play an important role in algorithmic information theory, see e.g. Li and Vitányi [14].

Schnorr [22], following Ville [29], characterized constructive null sets in terms of martingales. A function $d:\{0,1\}^{*} \rightarrow[0, \infty)$ is a martingale if for every $w \in\{0,1\}^{*}, d$ satisfies the averaging condition

$$
2 d(w)=d(w 0)+d(w 1),
$$

and $d$ is a supermartingale if it satisfies

$$
2 d(w) \geq d(w 0)+d(w 1) .
$$

The success set of $d$ is

$$
S^{\infty}[d]=\left\{A \in \mathbf{C} \mid \limsup _{n \rightarrow \infty} d(A\lceil n)=\infty\},\right.
$$

i.e., it is the set of all sequences on which $d$ has unbounded value. We say that $d$ succeeds on a class $\mathcal{A} \subseteq \mathbf{C}$ if $\mathcal{A} \subseteq S^{\infty}[d]$.

Ville [29] proved that a set $\mathcal{A} \subseteq \mathbf{C}$ has Lebesgue measure 0 if and only if there is a martingale $d$ that succeeds on $\mathcal{A}$. Schnorr [22] showed that $\mathcal{A}$ is constructively null if and only if $d$ can be chosen to be lower semicomputable, that is, if $d$ can be computably approximated from below. We call such a $d$ constructive.

Martin-Löf [17] proved that there is a universal constructive null set. That is, he proved that there is a Martin-Löf test $\left\{\mathcal{U}_{i}\right\}_{i}$ such that for every other test $\left\{\mathcal{V}_{i}\right\}$ it holds that $\bigcap_{i} \mathcal{V}_{i} \subseteq \bigcap_{i} \mathcal{U}_{i}$. By Schnorr's analysis this implies that there is also a universal constructive supermartingale $\mathbf{d}$. That is, for any constructive supermartingale $d^{\prime}$ there is a $c>0$ such that $\mathbf{d}(w) \geq c d^{\prime}(w)$ for all $w \in\{0,1\}^{*}$. We will use this universal supermartingale in section 4 . We denote the complement of $S^{\infty}[\mathbf{d}]$ by RAND, so that RAND consists of all the Martin-Löf random sequences.

\subsection{Schnorr Randomness}

Schnorr [22] criticized the notion of constructive null for an actual lack of constructiveness, and introduced the more constructive notion of a Schnorr null set, which is defined by requiring that the measure of the levels $\mathcal{U}_{i}$ in a Martin-Löf test be computably approximable to within any given precision. It is easy to see that this is equivalent to the following: $\mathcal{A}$ is Schnorr null if $\mathcal{A} \subseteq \bigcap_{i} \mathcal{U}_{i}$, 
where $\left\{\mathcal{U}_{i}\right\}_{i \in \mathbb{N}}$ is uniformly c.e. such that $\mu\left(\mathcal{U}_{i}\right)=2^{-i}$. The sequence $\left\{\mathcal{U}_{i}\right\}_{i \in \mathbb{N}}$ is called a $S$ chnorr test.

Following Schnorr [22], we call an unbounded nondecreasing function $h:\{0,1\}^{*} \rightarrow\{0,1\}^{*}$ an order. (N.B. An "Ordnungsfunktion" in Schnorr's terminology is always computable, whereas we prefer to leave the complexity of orders unspecified in general.) For any order $h$ and martingale $d$, we define the order $h$ success set of $d$ as

$$
S^{h}[d]=\left\{A \in \mathbf{C} \mid \limsup _{n \rightarrow \infty} \frac{d(A\lceil n)}{h(n)} \geq 1\right\} .
$$

Schnorr pointed out that the rate of success of a constructive martingale $d$ can be so slow that it cannot be computably detected. Thus rather than working with constructive null sets of the form $S^{\infty}[d]$ with $d$ constructive, he worked with null sets of the form $S^{h}[d]$, where both $d$ and $h$ are computable. He proved that a set $\mathcal{A}$ is Schnorr null if and only if it is included in a null set of the form $S^{h}[d]$, with $d$ and $h$ computable.

A sequence $A \in \mathbf{C}$ is Schnorr random if $\{A\}$ is not Schnorr null. This is related the notion of computable randomness. A sequence $A$ is computably random if for every computable martingale $d, A \notin S^{\infty}[d]$.

We write RAND Schnorr $_{\text {for the class of all Schnorr random sequences and RAND }}$ comp for the class of all computably random sequences. By definition we have that

$$
\mathrm{RAND} \subseteq \mathrm{RAND}_{\text {comp }} \subseteq \mathrm{RAND}_{\text {Schnorr }} .
$$

The first inclusion was proved strict by Schnorr [22] and the second inclusion was proved strict by Wang [32].

\subsection{Constructive Dimension}

Hausdorff [6] introduced the concept of null covers that "succeed exponentially fast" to define what is now commonly called Hausdorff dimension, the most widely used dimension in fractal geometry. Basically, this notion allows one to discern structure in classes of measure zero, and to calibrate them. As for constructive measure, already Schnorr (see Theorem 5.1) drew special attention to null sets of "exponential order", although he did not make an explicit connection to Hausdorff dimension.

Lutz $[15,16]$ gave a characterization of Hausdorff dimension in terms of gales, which are a generalization of martingales. Let $s \in[0, \infty)$. An $s$-gale is a function $d:\{0,1\}^{*} \rightarrow[0, \infty)$ that satisfies the averaging condition

$$
2^{s} d(w)=d(w 0)+d(w 1)
$$

for every $w \in\{0,1\}^{*}$. Similarly, $d$ is an s-supergale if (2.1) holds with $\geq$ instead of equality. The success set $S^{\infty}[d]$ is defined exactly as was done for martingales above. Lutz showed that for any class $\mathcal{A} \subseteq \mathbf{C}$, the Hausdorff dimension of $\mathcal{A}$ is

$$
\operatorname{dim}_{\mathrm{H}}(\mathcal{A})=\inf \left\{\begin{array}{l|l}
s \mid \begin{array}{l}
\text { there exists an s-gale } \\
d \text { for which } \mathcal{A} \subseteq S^{\infty}[d]
\end{array}
\end{array}\right\} .
$$

Lutz [16] effectivized this characterization to define the constructive dimensions of sets and sequences. An $s$-(super)gale is called constructive if it is lower semicomputable. The constructive dimension of a class $\mathcal{A} \subseteq \mathbf{C}$ is

$$
\operatorname{cdim}(\mathcal{A})=\inf \left\{\begin{array}{l|l}
s & \begin{array}{l}
\text { there exists a constructive s-gale } \\
d \text { for which } \mathcal{A} \subseteq S^{\infty}[d]
\end{array}
\end{array}\right\}
$$


and the constructive dimension of an individual sequence $A \in \mathbf{C}$ is

$$
\operatorname{dim}(A)=\operatorname{cdim}(\{A\})
$$

(Supergales can be equivalently used in place of gales in both $(2.2)$ and $(2.3)[15,9,4]$.)

Constructive dimension has some remarkable properties. For example, Lutz [16] showed that for any class $\mathcal{A}$,

$$
\operatorname{cdim}(\mathcal{A})=\sup _{A \in \mathcal{A}} \operatorname{dim}(A) .
$$

Also, Mayordomo [18] established a strong connection with Kolmogorov complexity: for any $A \in \mathbf{C}$,

$$
\operatorname{dim}(A)=\liminf _{n \rightarrow \infty} \frac{K(A\lceil n)}{n}
$$

where $K(A\lceil n)$ is the size of the smallest program that causes a fixed universal self-delimiting Turing machine to output the first $n$ bits of $A$. (For comments on the relation of this result to earlier results, see the note [26] by Staiger and section 6 of [16]. For more details on Kolmogorov complexity, we refer to [14].)

One can also characterize constructive dimension using the Schnorr null sets (see Section 2.2) of exponential order. The following proposition was observed by several authors, including those of $[1,27]$.

Proposition 2.1. Let $\mathbf{d}$ be the universal constructive supermartingale. For any $\mathcal{A} \subseteq \mathbf{C}$,

$$
\left.\operatorname{cdim}(\mathcal{A})=\inf \left\{s \in \mathbb{Q}: \mathcal{A} \subseteq S^{2^{(1-s) n}}[\mathbf{d}]\right)\right\} .
$$

\subsection{Constructive Strong Dimension}

More recently, Athreya, Hitchcock, Lutz, and Mayordomo [2] also characterized packing dimension, another important fractal dimension, in terms of gales. For this, the notion of strong success of an $s$-gale $d$ was introduced. The strong success set of $d$ is

$$
S_{\mathrm{str}}^{\infty}[d]=\left\{A \in \mathbf{C} \mid \liminf _{n \rightarrow \infty} d(A\lceil n)=\infty\} .\right.
$$

Analogously to what was done for Hausdorff dimension, packing dimension can be characterized using strong success sets of gales. Effectivizing this in the same way leads to the definition of the constructive strong dimension of a class $\mathcal{A} \subseteq \mathbf{C}$ as

$$
\operatorname{cDim}(\mathcal{A})=\inf \left\{\begin{array}{l|l}
s \mid \begin{array}{l}
\text { there exists a constructive } s \text {-gale } \\
d \text { for which } \mathcal{A} \subseteq S_{\text {str }}^{\infty}[d]
\end{array}
\end{array}\right\} .
$$

The constructive strong dimension of a sequence $A \in \mathbf{C}$ is

$$
\operatorname{Dim}(A)=\operatorname{cDim}(\{A\}) .
$$

A pointwise stability property analogous to (2.4) also holds for strong dimension, as well as a Kolmogorov complexity characterization [2]:

$$
\operatorname{Dim}(A)=\limsup _{n \rightarrow \infty} \frac{K(A\lceil n)}{n}
$$

for any $A \in \mathbf{C}$. 


\section{Borel Hierarchies}

$\boldsymbol{\Sigma}_{n}^{0}$ and $\boldsymbol{\Pi}_{n}^{0}$ denote the levels of the Borel hierarchy for subsets of Cantor space. The levels of the arithmetical hierarchy (the corresponding effective hierarchy for sets of reals) are denoted by $\Sigma_{n}^{0}$ and $\Pi_{n}^{0}$.

We will also make use of the following more general hierarchy definition.

Definition. Let $\mathcal{P}$ be a class of predicates, let $n \geq 1$, and let $\mathcal{X} \subseteq \mathbf{C}$.

- $\mathcal{X} \in \Sigma_{n}^{0}[\mathcal{P}]$ if for some predicate $P \in \mathcal{P}$,

$$
A \in \mathcal{X} \Longleftrightarrow\left(\exists k_{n}\right)\left(\forall k_{n-1}\right) \cdots\left(Q k_{1}\right) P\left(k_{n}, \ldots, k_{2}, A\left\lceil k_{1}\right),\right.
$$

where $Q=\exists$ if $n$ is odd and $Q=\forall$ if $n$ is even.

- $\mathcal{X} \in \Pi_{n}^{0}[\mathcal{P}]$ if for some predicate $P \in \mathcal{P}$,

$$
A \in \mathcal{X} \Longleftrightarrow\left(\forall k_{n}\right)\left(\exists k_{n-1}\right) \cdots\left(Q k_{1}\right) P\left(k_{n}, \ldots, k_{2}, A\left\lceil k_{1}\right),\right.
$$

where $Q=\forall$ if $n$ is odd and $Q=\exists$ if $n$ is even.

If we take $\mathcal{P}$ to be $\Delta_{1}^{0}$ (decidable), then the above definition is equivalent to the standard arithmetical hierarchy of reals, that is

$$
\Sigma_{n}^{0}=\Sigma_{n}^{0}\left[\Delta_{1}^{0}\right]
$$

and

$$
\Pi_{n}^{0}=\Pi_{n}^{0}\left[\Delta_{1}^{0}\right]
$$

hold for all $n$. Also, if ALL is the class of all predicates, then we obtain the classical Borel hierarchy:

$$
\Sigma_{n}^{0}=\Sigma_{n}^{0}[\mathrm{ALL}]
$$

and

$$
\Pi_{n}^{0}=\Pi_{n}^{0}[\mathrm{ALL}] .
$$

In this paper, we will also be interested in the cases where $\mathcal{P}$ is $\Sigma_{1}^{0}$ (computably enumerable) or $\Pi_{1}^{0}$ (co-c.e.). In some cases, the classes in the generalized hierarchy using these sets of predicates are no different than the standard arithmetical hierarchy classes. If $n$ is odd, then $\Sigma_{n}^{0}=\Sigma_{n}^{0}\left[\Sigma_{1}^{0}\right]$ as the existential quantifier in the $\Sigma_{1}^{0}$ predicate can be absorbed into the last quantifier in the definition of $\Sigma_{n}^{0}\left[\Delta_{1}^{0}\right]=\Sigma_{n}^{0}$. Analogously, $\Pi_{n}^{0}=\Pi_{n}^{0}\left[\Pi_{1}^{0}\right]$ for odd $n$, and for even $n$ we have $\Sigma_{n}^{0}=\Sigma_{n}^{0}\left[\Pi_{1}^{0}\right]$ and $\Pi_{n}^{0}=\Pi_{n}^{0}\left[\Sigma_{1}^{0}\right]$. On the other hand, using the complementary set of predicates defines an effective hierarchy that is distinct from and interleaved with the arithmetical hierarchy.

Proposition 3.1. $\quad$ 1. If $n$ is odd, then

$$
\Sigma_{n}^{0} \subsetneq \Sigma_{n}^{0}\left[\Pi_{1}^{0}\right] \subsetneq \Sigma_{n+1}^{0}
$$

and

$$
\Pi_{n}^{0} \subsetneq \Pi_{n}^{0}\left[\Sigma_{1}^{0}\right] \subsetneq \Pi_{n+1}^{0}
$$

2. If $n$ is even, then

$$
\Sigma_{n}^{0} \subsetneq \Sigma_{n}^{0}\left[\Sigma_{1}^{0}\right] \subsetneq \Sigma_{n+1}^{0}
$$

and

$$
\Pi_{n}^{0} \subsetneq \Pi_{n}^{0}\left[\Pi_{1}^{0}\right] \subsetneq \Pi_{n+1}^{0} .
$$


Proof. We only show $\Sigma_{n}^{0} \subsetneq \Sigma_{n}^{0}\left[\Pi_{1}^{0}\right] \subsetneq \Sigma_{n+1}^{0}$ for odd $n$; the arguments for the other statements are analogous.

The inclusion $\Sigma_{n}^{0} \subseteq \Sigma_{n}^{0}\left[\Pi_{1}^{0}\right]$ is obvious. To show that it is proper, let $P$ be a predicate that is complete for the class of $\Pi_{n}^{0}$ predicates. Then there is a decidable predicate $R$ such that

$$
P(j) \Longleftrightarrow\left(\forall k_{n}\right)\left(\exists k_{n-1}\right) \cdots\left(\forall k_{1}\right) R\left(j, k_{n}, \cdots, k_{1}\right)
$$

Define $\mathcal{X} \subseteq \mathbf{C}$ as

$$
\mathcal{X}=\bigcup_{j \in P} 0^{j} 1 \mathbf{C} .
$$

Let $f: \omega \times \omega \rightarrow \omega$ be a computable bijection and let $f_{1}: \omega \rightarrow \omega$ such that $f_{1}(f(i, j))=i$ for all $i, j$. Assume $n \geq 3$. Then $\mathcal{X} \in \Sigma_{n}^{0}\left[\Pi_{1}^{0}\right]$ as we have

$$
\begin{aligned}
S \in \mathcal{X} & \Longleftrightarrow(\exists j) P(j) \text { and } 0^{j} 1 \sqsubseteq S \\
& \Longleftrightarrow(\exists j)\left(\forall k_{n}\right)\left(\exists k_{n-1}\right) \cdots\left(\forall k_{1}\right) R\left(j, k_{n}, \cdots, k_{1}\right) \text { and } 0^{j} 1 \sqsubseteq S \\
& \Longleftrightarrow(\exists j)\left(\forall k_{n}\right)\left(\exists k_{n-1}\right) \cdots\left(\forall k_{3}\right)(\exists l) T\left(j, k_{n}, \cdots, k_{3}, S \uparrow l\right),
\end{aligned}
$$

where $T$ is the $\Pi_{1}^{0}$ predicate defined by

$$
T\left(j, k_{n}, \cdots, k_{3}, w\right) \Longleftrightarrow\left(\forall k_{1}\right) R\left(j, k_{n}, \cdots, k_{3}, f_{1}(|w|), k_{1}\right) \text { and } 0^{j} 1 \sqsubseteq w .
$$

For the case $n=1$, we have $\mathcal{X} \in \Sigma_{1}^{0}\left[\Pi_{1}^{0}\right]$ because

$$
\begin{aligned}
S \in \mathcal{X} & \Longleftrightarrow(\exists j)\left(\forall k_{1}\right) R\left(j, k_{1}\right) \text { and } 0^{j} 1 \sqsubseteq S \\
& \Longleftrightarrow\left(\exists j^{\prime}\right) T\left(S \uparrow j^{\prime}\right),
\end{aligned}
$$

where $T$ is the $\Pi_{1}^{0}$ predicate defined by

$$
T(w) \Longleftrightarrow\left(\forall k_{1}\right) R\left(f_{1}\left(j^{\prime}\right), k_{1}\right) \text { and } 0^{f_{1}\left(j^{\prime}\right)} 1 \sqsubseteq w .
$$

Now suppose that $\mathcal{X} \in \Sigma_{n}^{0}$. Then for some decidable predicate $U$,

$$
S \in X \Longleftrightarrow\left(\exists k_{n}\right)\left(\forall k_{n-1}\right) \cdots\left(\exists k_{1}\right) U\left(k_{n}, \cdots, k_{2}, S \uparrow k_{1}\right) .
$$

We then have

$$
\begin{aligned}
j \in P & \Longleftrightarrow 0^{j} 1 \mathbf{C} \subseteq \mathcal{X} \\
& \Longleftrightarrow 0^{j} 10^{\infty} \in \mathcal{X} \\
& \Longleftrightarrow\left(\exists k_{n}\right)\left(\forall k_{n-1}\right) \cdots\left(\exists k_{1}\right) U\left(k_{n}, \cdots, k_{2}, 0^{j} 10^{\infty}\left\lceil k_{1}\right),\right.
\end{aligned}
$$

so $P$ is a $\Sigma_{n}^{0}$ predicate, which contradicts its $\Pi_{n}^{0}$-completeness. Therefore $\mathcal{X} \notin \Sigma_{n}^{0}$ and we have established $\Sigma_{n}^{0} \subsetneq \Sigma_{n}^{0}\left[\Pi_{1}^{0}\right]$.

To see that $\Sigma_{n}^{0}\left[\Pi_{1}^{0}\right] \subseteq \Sigma_{n+1}^{0}$, let $\mathcal{X} \in \Sigma_{n}^{0}\left[\Pi_{1}^{0}\right]$. Then there is a $\Pi_{1}^{0}$ predicate $P$ such that

$$
S \in \mathcal{X} \Longleftrightarrow\left(\exists k_{n}\right) \cdots\left(\exists k_{1}\right) P\left(k_{n}, \ldots, S \uparrow k_{1}\right) .
$$

Let $R$ be decidable such that

$$
P\left(k_{n}, \ldots, w\right) \Longleftrightarrow(\forall j) R\left(k_{n}, \ldots, w, j\right) .
$$


Define $R^{\prime}$ by

$$
R^{\prime}\left(k_{n}, \ldots, k_{1}, w\right) \Longleftrightarrow R\left(k_{n}, \ldots, k_{2}, w\left\lceil k_{1},|w|\right) \text { or }|w|<k_{1} .\right.
$$

Then

$$
S \in \mathcal{X} \Longleftrightarrow\left(\exists k_{n}\right) \cdots\left(\exists k_{1}\right)(\forall l) R^{\prime}\left(k_{n}, \ldots, k_{1}, S \uparrow l\right),
$$

so $\mathcal{X} \in \Sigma_{n+1}^{0}$. That $\Sigma_{n}^{0}\left[\Pi_{1}^{0}\right] \neq \Sigma_{n+1}^{0}$ follows from the facts $\Sigma_{n+1}^{0}-\Sigma_{n}^{0} \neq \emptyset$ (see [19,3E.8]) and $\Sigma_{n}^{0}\left[\Pi_{1}^{0}\right] \subseteq \Sigma_{n}^{0}$.

The next proposition shows that there are no unexpected inclusions:

Proposition 3.2. $\quad$ 1. If $n$ is odd, then

$$
\Sigma_{n}^{0} \nsubseteq \nsubseteq \Pi_{n}^{0}\left[\Sigma_{1}^{0}\right] \nsubseteq \Sigma_{n+1}^{0}
$$

and

$$
\Pi_{n}^{0} \not \subset \Sigma_{n}^{0}\left[\Pi_{1}^{0}\right] \nsubseteq \subseteq \Pi_{n+1}^{0}
$$

2. If $n$ is even, then

$$
\Sigma_{n}^{0} \not \subset \Pi_{n}^{0}\left[\Pi_{1}^{0}\right] \nsubseteq \Sigma_{n+1}^{0}
$$

and

$$
\Pi_{n}^{0} \not \subset \Sigma_{n}^{0}\left[\Sigma_{1}^{0}\right] \nsubseteq \Pi_{n+1}^{0}
$$

Proof. The noninclusions on the left side all follow from Borel considerations. E.g. for the noninclusion $\Pi_{n}^{0} \nsubseteq \Sigma_{n}^{0}\left[\Pi_{1}^{0}\right]$ take any $\Pi_{n}^{0}$-class that is not in $\boldsymbol{\Sigma}_{n}^{0}$.

The noninclusions on the right side can all be proved by direct diagonalization. As an example we prove that $\Sigma_{1}^{0}\left[\Pi_{1}^{0}\right] \nsubseteq \Pi_{2}^{0}$. The proof easily generalizes to the higher levels. The proof is a fairly straightforward diagonalization against all possible $\Pi_{2}^{0}$-definitions, although the details are a bit cumbersome. Let $R_{i}, i \in \omega$, be a computable list of all partial computable predicates. We define a class $\mathcal{X} \in \Sigma_{1}^{0}\left[\Pi_{1}^{0}\right]$ such that for all $i$ there is $X \sqsupset 0^{i} 1$ such that

$$
X \in(\mathcal{X} \backslash \mathcal{Y}) \cup(\mathcal{Y} \backslash \mathcal{X})
$$

where $\mathcal{Y}=\left\{X: \forall n \exists m R_{i}(n, X \uparrow m)\right\}$. So the definition of $\mathcal{X}$ in the interval above the string $0^{i} 1$ will make sure that $\mathcal{X}$ is not $\Pi_{2}^{0}$-defined by $R_{i}$.

For the definition of $\mathcal{X}$ we will need a uniform sequence of $\Pi_{1}^{0}$-sets of strings $P_{n}^{i}$. We start by defining $\operatorname{dom}\left(P_{n}^{i}\right)$ for each $i$ and $n$ such that

- $\operatorname{dom}\left(P_{n}^{i}\right)$ is a computable subset of $\left\{\sigma 1: \sigma \sqsupseteq 0^{i} 1\right\}$,

- $\operatorname{dom}\left(P_{n}^{i}\right)$ is dense above the string $0^{i} 1$, i.e. for all $\tau \sqsupseteq 0^{i} 1$ there is $\sigma \sqsupseteq \tau$ with $\sigma \in \operatorname{dom}\left(P_{n}^{i}\right)$,

- $\operatorname{dom}\left(P_{n}^{i}\right) \cap \operatorname{dom}\left(P_{m}^{i}\right)=\emptyset$ if $n \neq m$.

Then we define $P_{n}^{i}$ by

- for every $\sigma 1 \in \operatorname{dom}\left(P_{n}^{i}\right)$,

$$
\sigma 1 \notin P_{n}^{i} \Longleftrightarrow(\exists \tau \sqsupseteq \sigma 1)(\exists m)\left[\tau \text { is of the form } \sigma 10^{k} \wedge R_{i}(n, \tau\lceil m)] .\right.
$$


It is easy to see that such a uniform sequence of $P_{n}^{i}$ 's exists. Now $\mathcal{X}$ is defined as

$$
\mathcal{X}=\left\{X:(\exists i)(\exists n)\left(\exists \sigma \in P_{n}^{i}\right)[\sigma \sqsubset X]\right\} .
$$

The idea is that to show that $R_{i}$ does not give a $\Pi_{2}^{0}$-definition of $\mathcal{X}$, we challenge it by choosing a string $\sigma 1 \in P_{n}^{i}$ and extend it by 0 's. Now if $R_{i}$ responds by providing us with an extension $\tau$ as in (3.2), we take this $\tau$ as an initial segment of our set $X$, which means that there is an $m$ such that $R_{i}\left(n, X\lceil m)\right.$, so that the condition $\left.\forall n \exists m R_{i}(n, X\rceil m\right)$ is verified for $n$. But by definition of $P_{n}^{i}$, as soon as the witness $m$ is found, the string $\sigma 1$ falls out of the set $P_{n}^{i}$, so we have mananged to keep $X$ outside of $\mathcal{X}$ while at the same time obtaining a piece of evidence that $\forall n \exists m R_{i}(n, X\lceil m)$. If on the other hand $R_{i}$ does not respond this means that $\sigma 1 \in P_{n}^{i}$, so $X$ will be in $\mathcal{X}$, but no extension $Y$ of $\sigma 1$ will satisfy $\forall n \exists m R_{i}(n, Y \uparrow m)$. So in both cases $X$ is a counterexample showing that $R_{i}$ does not define $\mathcal{X}$.

We now give the formal construction. Fix $i$. We construct $X$ as in (3.1) by a finite extension construction. Let $X_{0}=0^{i} 1$. At stage $s$ of the construction we are given $X_{s}$, no initial segment of which is in any $P_{n}^{i}$, and such that $(\forall n \leq s)\left(\exists m \leq\left|X_{s}\right|\right)\left[R_{i}\left(n, X_{s}\lceil m)\right]\right.$. Choose $\sigma 1 \in \operatorname{dom}\left(P_{s+1}^{i}\right)$ such that $\sigma 1 \sqsupset X_{s}$.

Case I. There exists a $\tau$ as in (3.2), with $n=s+1$. Define $X_{s+1}=\tau$ and go to the next stage of the construction.

Case II. There does not exist such a $\tau$. Then define $X=X_{s}{ }^{\wedge} 0^{\omega}$ and end the construction.

To verify that $X=\bigcup_{s} X_{s}$ thus constructed satisfies (3.1), note that if there is a stage where Case II obtains, then the string $\sigma 1$ chosen at that stage is in $P_{s}^{i}$ and proves that $X \in \mathcal{X}$, whereas no extension $Y$ of $\sigma 1$ satisfies $\exists m R_{i}(n, Y \uparrow m)$. So in this case we are done. If on the other hand at every stage of the construction Case I obtains, then for every $\sigma 1$ chosen at any stage $s$ we have $\sigma 1 \notin P_{s}^{i}$, and hence $X \notin \mathcal{X}$ since apart from the 1's in $\sigma 1$ the string $X$ only contains 0's, and $\operatorname{dom}\left(P_{n}^{i}\right) \subseteq\left\{\sigma 1: \sigma \sqsupseteq 0^{i} 1\right\}$. But also at every stage $s$ a new witness $m$ is found such that $R_{i}\left(s, X\lceil m)\right.$, hence $\forall s \exists m R_{i}(s, X\lceil m)$, and again $X$ satisfies (3.1).

Intuitively, the classes $\Sigma_{1}^{0}\left[\Pi_{1}^{0}\right], \Pi_{1}^{0}\left[\Sigma_{1}^{0}\right], \Sigma_{2}^{0}\left[\Sigma_{1}^{0}\right], \Pi_{2}^{0}\left[\Pi_{1}^{0}\right], \ldots$ are slightly more powerful than their respective counterparts in the arithmetical hierarchy because they use one additional quantifier that is limited to the predicate. We now give a simple example of a class that is best classified in this hierarchy: the class of 1 -generic sequences.

Proposition 3.3. The class of all 1-generic sequences is $\Pi_{2}^{0}\left[\Pi_{1}^{0}\right]$ but not $\Sigma_{3}^{0}$. It is also not $\boldsymbol{\Sigma}_{2}^{0}$.

Proof. Recall that a sequence $X \in \mathbf{C}$ is 1-generic (see e.g. Jockusch [10]) if

$$
(\forall e)(\exists \sigma \sqsubset X)\left[\{e\}^{\sigma}(e) \downarrow \vee(\forall \tau \sqsupset \sigma)\left[\{e\}^{\tau}(e) \uparrow\right]\right]
$$

From this definition it is immediate that the class $\mathcal{G}=\{X \mid X$ is 1 -generic $\}$ is in $\Pi_{2}^{0}\left[\Pi_{1}^{0}\right]$. To show that $\mathcal{G}$ is not $\Sigma_{3}^{0}$, suppose that it is. Then there is a uniform sequence of $\Sigma_{1}^{0}$-classes $\mathcal{O}_{n, m}$ such that $\mathcal{G}=\bigcup_{n} \bigcap_{m} \mathcal{O}_{n, m}$. Without loss of generality $\mathcal{O}_{n, m} \supseteq \mathcal{O}_{n, m+1}$ for all $n, m$. Now $\mathcal{G}$ is comeager, so there is $n$ such that $\bigcap_{m} \mathcal{O}_{n, m}$ is not nowhere dense, hence dense in some interval $C_{\sigma}$. Then every $\mathcal{O}_{n, m}, m \in \mathbb{N}$, is dense in $C_{\sigma}$. Now it is easy to construct, using a computable finite extension construction, a computable sequence (starting with $\sigma$ ) in $\bigcap_{m} \mathcal{O}_{n, m}$, contradicting that 1-generic sets are noncomputable.

That the 1-generic sets are not $\boldsymbol{\Sigma}_{\mathbf{2}}^{\mathbf{0}}$ follows quickly from Lemma 4.3 below, noting again that the 1-generic sets are a comeager class. 
Staiger has pointed out to us that the class $\Pi_{1}^{0}\left[\Sigma_{1}^{0}\right]$ already occured under a different guise in [25] where it was called $\mathfrak{P}$, and several presentations were proven to be equivalent to it. The following definitions are contained in [24]. Let $W$ be any set of initial segments. Define

$$
\begin{aligned}
\lim W & =\left\{A \in 2^{\omega}: \forall \sigma \sqsubset A(\sigma \in W)\right\}, \\
W^{\sigma} & =\left\{A \in 2^{\omega}: \forall^{\infty} \sigma \sqsubset A(\sigma \in W)\right\} .
\end{aligned}
$$

Staiger proved that the classes in $\Pi_{1}^{0}\left[\Sigma_{1}^{0}\right]$ are those of the form $\lim W$, for $W \in \Sigma_{1}^{0}$, and the classes in $\Sigma_{2}^{0}\left[\Sigma_{1}^{0}\right]$ are those of the form $W^{\sigma}$, for $W \in \Sigma_{1}^{0}$.

\section{Classification of $\mathrm{DIM}^{\alpha}$ and $\mathrm{DIM}_{\mathrm{str}}^{\alpha}$}

In this section we investigate the arithmetical complexity of the following dimension and strong dimension classes.

$$
\begin{aligned}
\operatorname{DIM}^{\alpha} & =\{A \in \mathbf{C} \mid \operatorname{dim}(A)=\alpha\} \\
\operatorname{DIM}^{\leq \alpha} & =\{A \in \mathbf{C} \mid \operatorname{dim}(A) \leq \alpha\} \\
\mathrm{DIM}^{\geq \alpha} & =\{A \in \mathbf{C} \mid \operatorname{dim}(A) \geq \alpha\} \\
\operatorname{DIM}_{\text {str }}^{\alpha} & =\{A \in \mathbf{C} \mid \operatorname{Dim}(A)=\alpha\} \\
\operatorname{DIM}_{\text {str }}^{\leq \alpha} & =\{A \in \mathbf{C} \mid \operatorname{Dim}(A) \leq \alpha\} \\
\operatorname{DIM}_{\text {str }}^{\geq \alpha} & =\{A \in \mathbf{C} \mid \operatorname{Dim}(A) \geq \alpha\}
\end{aligned}
$$

Let $\alpha \in[0,1]$ be $\Delta_{2}^{0}$-computable. For any such $\alpha$, it is well known that there is a computable function $\hat{\alpha}: \mathbb{N} \rightarrow \mathbb{Q}$ such that $\lim _{n \rightarrow \infty} \hat{\alpha}(n)=\alpha$. Using (2.5), we have

$$
\begin{aligned}
\operatorname{dim}(X) \leq \alpha & \Longleftrightarrow \liminf _{n \rightarrow \infty} \frac{K(X\lceil n)}{n} \leq \alpha \\
& \Longleftrightarrow \quad(\forall k)(\forall N)(\exists n \geq N) K(X\lceil n)<(\hat{\alpha}(n)+1 / k) n,
\end{aligned}
$$

so $\mathrm{DIM}^{\leq \alpha}$ is a $\Pi_{2}^{0}$-class. Also,

$$
\begin{aligned}
\operatorname{dim}(X) \geq \alpha & \Longleftrightarrow \liminf _{n \rightarrow \infty} \frac{K(X\lceil n)}{n} \geq \alpha \\
& \Longleftrightarrow(\forall k)(\exists N)(\forall n \geq N) K(X\lceil n)>(\hat{\alpha}(N)-1 / k) n,
\end{aligned}
$$

so $\mathrm{DIM}^{\geq \alpha}$ is a $\Pi_{3}^{0}$-class. Therefore we have the following.

Proposition 4.1. 1. The class $\mathrm{DIM}^{0}$ is $\Pi_{2}^{0}$.

2. For all $\Delta_{2}^{0}$-computable $\alpha \in(0,1]$, $\mathrm{DIM}^{\alpha}$ is a $\Pi_{3}^{0}$-class.

3. For arbitrary $\alpha \in(0,1], \mathrm{DIM}^{\alpha}$ is a $\boldsymbol{\Pi}_{3}^{0}$-class.

The situation is slightly more complicated for strong dimension. By (2.6), we have

$$
\begin{aligned}
\operatorname{Dim}(X) \leq \alpha & \Longleftrightarrow \quad \limsup _{n \rightarrow \infty} \frac{K(X \uparrow n)}{n} \leq \alpha \\
& \Longleftrightarrow \quad(\forall k)(\exists N)(\forall n \geq N) K(X \uparrow n)<(\hat{\alpha}(N)+1 / k) n \\
& \Longleftrightarrow \quad(\forall k)(\exists N)(\forall n \geq N)(\exists\langle\pi, t\rangle)|\pi|<(\hat{\alpha}(N)+1 / k) n \\
& \quad \text { and } U(\pi)=X\lceil n \text { in } \leq t \text { computation steps, }
\end{aligned}
$$


where $U$ is the fixed universal self-delimiting Turing machine used to define $K$. From this it is clear that $\operatorname{DIM}_{\text {str }}^{\leq \alpha} \in \Pi_{4}^{0}$. However, the " $(\exists\langle\pi, t\rangle)$ " quantifier is local to the defining predicate, so we have $\operatorname{DIM}_{\mathrm{str}}^{\leq \alpha} \in \Pi_{3}^{0}$, and in fact, it is a $\Pi_{3}^{0}\left[\Sigma_{1}^{0}\right]$-class. Also,

$$
\begin{aligned}
\operatorname{Dim}(X) \geq \alpha & \Longleftrightarrow \limsup _{n \rightarrow \infty} \frac{K(X\lceil n)}{n} \geq \alpha \\
& \Longleftrightarrow(\forall k)(\forall N)(\exists n \geq N) K(X\lceil n)>(\hat{\alpha}(n)-1 / k) n
\end{aligned}
$$

so $\mathrm{DIM}_{\mathrm{str}}^{\geq \alpha}$ is a $\Pi_{2}^{0}\left[\Pi_{1}^{0}\right]$-class. This establishes the following analogue of Proposition 4.1.

Proposition 4.2. 1. The class $\mathrm{DIM}_{\mathrm{str}}^{1}$ is $\Pi_{2}^{0}\left[\Pi_{1}^{0}\right]$.

2. For all $\Delta_{2}^{0}$-computable $\alpha \in[0,1)$, $\operatorname{DIM}_{\mathrm{str}}^{\alpha}$ is a $\Pi_{3}^{0}\left[\Sigma_{1}^{0}\right]$-class.

3. For arbitrary $\alpha \in[0,1), \mathrm{DIM}_{\mathrm{str}}^{\alpha}$ is a $\Pi_{3}^{0}$-class.

In the remainder of this section we prove that the classifications in Propositions 4.1 and 4.2 cannot be improved in their respective hierarchies.

\subsection{Category Methods}

Recall that a class $\mathcal{X}$ is meager if it is included in a countable union of nowhere dense subsets of $\mathbf{C}$, and comeager if its complement $\overline{\mathcal{X}}$ is meager. The following lemma (implicit in Rogers [21, p341]) will be useful.

Lemma 4.3. If $\mathcal{X} \in \Sigma_{2}^{0}$ and $\overline{\mathcal{X}}$ is dense then $\mathcal{X}$ is meager.

Proof. Suppose that $\mathcal{X}=\bigcup_{n} \mathcal{X}_{n}, \mathcal{X}_{n}$ closed. Since $\overline{\mathcal{X}}$ is dense, $\mathcal{X}_{n}$ contains no basic open set, hence $\mathcal{X}_{n}$ is nondense (i.e. its closure contains no basic open set), and $\mathcal{X}$ is a countable union of nondense sets.

As a warm-up we give a short proof of Shoenfield's result that the class of computable sequences is not a $\Pi_{3}^{0}$-class.

Theorem 4.4. (Shoenfield $[21, \mathrm{p} 346])$ The class $\Delta_{1}^{0}$ of computable sets is a $\Sigma_{2}^{0}\left[\Sigma_{1}^{0}\right]$-class, but it is not a $\Pi_{3}^{0}$-class. It is also not a $\Pi_{2}^{0}$-class.

Proof. Clearly $\Delta_{1}^{0} \in \Sigma_{2}^{0}\left[\Sigma_{1}^{0}\right]$. Suppose for a contradiction that $\overline{\Delta_{1}^{0}}$ is $\Sigma_{3}^{0}$. Then there is a uniform sequence of $\Sigma_{1}^{0}$-classes $\mathcal{O}_{n, m}$ such that $\overline{\Delta_{1}^{0}}=\bigcup_{n} \bigcap_{m} \mathcal{O}_{n, m}$. Without loss of generality $\mathcal{O}_{n, m} \supseteq$ $\mathcal{O}_{n, m+1}$ for all $n, m$. Now $\Delta_{1}^{0}$ is meager because it is countable, so $\overline{\Delta_{1}^{0}}$ is comeager, so there is an $n$ such that $\bigcap_{m} \mathcal{O}_{n, m}$ is not nowhere dense, hence dense in some interval $C_{\sigma}$. Then every $\mathcal{O}_{n, m}$, $m \in \mathbb{N}$, is dense in $C_{\sigma}$. Now it is easy to construct a computable sequence (starting with $\sigma$ ) in $\bigcap_{m} \mathcal{O}_{n, m}$, contradicting that $\bigcap_{m} \mathcal{O}_{n, m} \subseteq \overline{\Delta_{1}^{0}}$.

That $\Delta_{1}^{0}$ is not $\Pi_{2}^{0}$ follows from Lemma 4.3 , since $\overline{\Delta_{1}^{0}}$ is comeager.

The class RAND of Martin-Löf random sets can easily be classified with category methods.

Theorem 4.5. (folk) RAND is a $\Sigma_{2}^{0}$-class, but it is not a $\Pi_{2}^{0}$-class.

Proof. This is analogous to the proof in Rogers [21, p 341] that $\{X: X$ finite $\}$ is a $\Sigma_{2}^{0}$-class but not a $\Pi_{2}^{0}$-class. Both RAND and its complement are dense, so by Lemma 4.3, RAND is meager. If RAND were a $\boldsymbol{\Pi}_{2}^{0}$-class, then again using Lemma 4.3 , its complement would also be meager. This contradicts the fact that $\mathbf{C}$ is not meager. 
As DIM $^{0}$ and DIM $_{\text {str }}^{1}$ are dense $\boldsymbol{\Pi}_{2}^{0}$-classes that have dense complements, an argument similar to the one used for Theorem 4.5 shows that they are not $\boldsymbol{\Sigma}_{2}^{0}$-classes.

Theorem 4.6. The classes $\mathrm{DIM}^{0}$ and $\mathrm{DIM}_{\mathrm{str}}^{1}$ are not $\boldsymbol{\Sigma}_{2}^{0}$-classes.

We now develop category methods for the other $\mathrm{DIM}^{\alpha}$ classes. For every rational $s$, define the computable order $h_{s}(n)=2^{(1-s) n}$. Let $\mathbf{d}$ be the optimal constructive supermartingale.

Lemma 4.7. For every rational $s \in(0,1), S^{h_{s}}[\mathbf{d}]$ is a comeager $\Pi_{2}^{0}$-class.

Proof. Notice that $\overline{S^{h_{s}}[\mathbf{d}]} \in \Sigma_{2}^{0}$ and $S^{h_{s}}[\mathbf{d}]$ is dense. Now apply Lemma 4.3.

Lemma 4.8. For all $\alpha \in(0,1], \mathrm{DIM}^{\alpha}$ is meager.

Proof. Let $s<\alpha$ be rational. Lutz [16] showed that $\mathbf{d}^{(s)}(w)=2^{(s-1)|w|} \mathbf{d}(w)$ is an optimal constructive $s$-supergale. It follows that for any $A \in \mathbf{C}, A \in S^{h_{s}}[\mathbf{d}] \Rightarrow \operatorname{dim}(S)<\alpha$. Therefore $\mathrm{DIM}^{\alpha} \subseteq \overline{S^{h_{s}}}$, so $\mathrm{DIM}^{\alpha}$ is meager by Lemma 4.7 .

Proposition 4.9. For all $\alpha \in(0,1]$, $\mathrm{DIM}^{\alpha}$ is not a $\Pi_{2}^{0}$-class.

Proof. If $\mathrm{DIM}^{\alpha} \in \Pi_{2}^{0}$, then Lemma 4.3 implies that $\mathrm{DIM}^{\alpha}$ is comeager, contradicting Lemma 4.8 .

To strengthen Proposition 4.9 to show that $\mathrm{DIM}^{\alpha}$ is not $\Sigma_{3}^{0}$, we now turn to Wadge reductions.

\subsection{Wadge Reductions}

Let $\mathcal{A}, \mathcal{B} \subseteq \mathbf{C}$. A Wadge reduction $[30,31,11]$ of $\mathcal{A}$ to $\mathcal{B}$ is a function $f: \mathbf{C} \rightarrow \mathbf{C}$ that is continuous and satisfies $\mathcal{A}=f^{-1}(\mathcal{B})$, i.e., $X \in \mathcal{A} \Longleftrightarrow f(X) \in \mathcal{B}$. We say that $\mathcal{B}$ is Wadge complete for a class $\boldsymbol{\Gamma}$ of subsets of $\mathbf{C}$ if $\mathcal{B} \in \boldsymbol{\Gamma}$ and every $\mathcal{A} \in \boldsymbol{\Gamma}$ Wadge reduces to $\mathcal{B}$. As the classes of the Borel hierarchy are closed under Wadge reductions, Wadge completeness can be used to properly identify the location of a subset of $\mathbf{C}$ in the hierarchy.

We now prove that $\mathrm{DIM}^{1}$ is Wadge complete for $\boldsymbol{\Pi}_{3}^{0}$. We will then give Wadge reductions from it to $\mathrm{DIM}^{\alpha}$ for the other values of $\alpha$.

Theorem 4.10. $\mathrm{DIM}^{1}$ is Wadge complete for $\boldsymbol{\Pi}_{3}^{0}$. Therefore $\mathrm{DIM}^{1}$ is not a $\boldsymbol{\Sigma}_{3}^{0}$-class, and in particular it is not a $\Sigma_{3}^{0}$-class.

Proof. One could prove this by reducing a known $\boldsymbol{\Pi}_{3}^{0}$-complete class to $\mathrm{DIM}^{1}$, e.g. the class of sets that have a limiting frequency of 1's that is 0 (this class was proved to be $\mathbf{\Pi}_{3}^{0}$-complete by Ki and Linton [12]), but it is just as easy to build a direct reduction from an arbitrary $\boldsymbol{\Pi}_{3}^{0}$-class.

Let $\mathbf{d}$ be the universal constructive supermartingale. Note that we have (cf. Proposition 2.1)

$$
S^{2^{n}}[\mathbf{d}] \subsetneq \ldots \subsetneq S^{2^{\frac{1}{k} n}}[\mathbf{d}] \subsetneq S^{2^{\frac{1}{k+1} n}}[\mathbf{d}] \subsetneq \ldots \subsetneq \mathrm{DIM}^{1} .
$$

Let $\bigcup_{k} \bigcap_{s} \mathcal{O}_{k, s}$ be a $\boldsymbol{\Sigma}_{3}^{0}$-class. Without loss of generality $\mathcal{O}_{k, s} \supseteq \mathcal{O}_{k, s+1}$ for all $k, s$. We define a continuous function $f: \mathbf{C} \rightarrow \mathbf{C}$ such that

$$
\forall k\left(X \in \bigcap_{s} \mathcal{O}_{k, s} \Longleftrightarrow f(X) \in S^{2^{\frac{1}{k} n}}[\mathbf{d}]\right)
$$


so that we have

$$
\begin{aligned}
X \notin \bigcup_{k} \bigcap_{s} \mathcal{O}_{k, s} & \Longleftrightarrow \forall k\left(f(X) \notin S^{2^{\frac{1}{k} n}}[\mathbf{d}]\right) \\
& \Longleftrightarrow f(X) \in \mathrm{DIM}^{1} .
\end{aligned}
$$

The image $Y=f(X)$ is defined in stages, $Y=\bigcup_{s} Y_{s}$.

At stage 0 we define $Y_{0}$ to be the empty sequence.

At stage $s>0$ we consider $X\left\lceil s\right.$, and for each $k$ we define $t_{k, s}$ to be the largest stage $t \leq s$ such that $X\left\lceil s \in \mathcal{O}_{k, t}\right.$. (Let $t_{k, s}=0$ if such a $t$ does not exist.) Define $k$ to be expansionary at stage $s$ if $t_{k, s-1}<t_{k, s}$. Now we let $k(s)=\min \{k: k$ is expansionary at $s\}$. There are two substages.

Substage (a). First consider all strings $\sigma$ extending $Y_{s-1}$ of minimal length with $\mathbf{d}(\sigma) \geq 2^{\frac{1}{k(s)}|\sigma|}$,

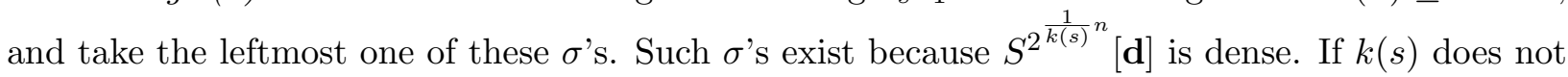
exist, let $\sigma=Y_{s-1}$.

Substage (b). Next consider all extensions $\tau \sqsupseteq \sigma$ of minimal length such that $\mathbf{d}(\tau\lceil i) \leq \mathbf{d}(\tau \uparrow$ $(i-1)$ ) for every $|\sigma|<i<|\tau|$, and $\mathbf{d}(\tau) \leq|\tau|$. Clearly such $\tau$ exist, by direct diagonalization against $\mathbf{d}$. Define $Y_{s}$ to be the leftmost of these $\tau$. This concludes the construction.

So $Y_{s}$ is defined by first building a piece of evidence $\sigma$ that $\mathbf{d}$ achieves growth rate $2^{\frac{1}{k(s)} n}$ on $Y$ and then slowing down the growth rate of $\mathbf{d}$ to the order $n$. Note that $f$ is continuous since the definition of the initial segment $Y_{s}$ depends only on the finite part $X\left\lceil s\right.$ of $X$. If $X \in \bigcup_{k} \bigcap_{s} \mathcal{O}_{k, s}$, then for the minimal $k$ such that $X \in \bigcap_{s} \mathcal{O}_{k, s}$, infinitely many pieces of evidence $\sigma$ witness that d achieves growth rate $2^{\frac{1}{k} n}$ on $Y$, so $Y \notin \mathrm{DIM}^{1}$. On the other hand, if $X \notin \bigcup_{k} \bigcap_{s} \mathcal{O}_{k, s}$ then for every $k$ only finitely often $\mathbf{d}\left(Y_{s}\right) \geq 2^{\frac{1}{k}\left|Y_{s}\right|}$ because in substage (a) the extension $\sigma$ is chosen to be of minimal length, so $Y \notin S_{h_{k}}[\mathbf{d}]$. Hence $Y \in \operatorname{DIM}^{1}$.

As RAND is a $\Sigma_{2}^{0}$-class, we have the following corollary (which can also be proved by a direct construction).

Corollary 4.11. (Lutz [16]) RAND is a proper subset of DIM ${ }^{1}$.

In order to establish the existence of $\Delta_{2}^{0}$-computable sequences of any $\Delta_{2}^{0}$-computable dimension $\alpha \in[0,1)$, Lutz [16] defined a dilution function $g_{\alpha}: \mathbf{C} \rightarrow \mathbf{C}$ that is computable and satisfies $\operatorname{dim}\left(g_{\alpha}(X)\right)=\alpha \cdot \operatorname{dim}(X)$ for all $X \in \mathbf{C}$. Applying this to any $\Delta_{2}^{0}$-computable Martin-Löf random sequence (which must have dimension 1) establishes the existence theorem. (We note that $g_{\alpha}(X)$ has the same Turing degree as $X$. Since by the Low Basis Theorem of Jockusch and Soare [20, Theorem V.5.32] there are Martin-Löf random sets of low degree, we immediately obtain that there are low sets of any $\Delta_{2}^{0}$-computable dimension $\alpha$.) As $g_{\alpha}$ is continuous, it is a Wadge reduction from $\mathrm{DIM}^{1}$ to DIM ${ }^{\alpha}$ if $\alpha>0$. Combining this with the previous theorem, we have that $\mathrm{DIM}^{\alpha}$ is Wadge complete for $\boldsymbol{\Pi}_{3}^{0}$ for all $\Delta_{2}^{0}$-computable $\alpha \in(0,1)$. We now give a similar dilution construction that will allow us to prove this for arbitrary $\alpha \in(0,1)$.

Let $X \in \mathbf{C}$ and let $\alpha \in(0,1)$. Write $X=x_{1} x_{2} x_{3} \ldots$ where $\left|x_{n}\right|=2 n-1$ for all $n$, noting that $\left|x_{1} \cdots x_{n}\right|=n^{2}$. For each $n$, let

$$
k_{n}=\left\lceil n \frac{1-\alpha}{\alpha}\right\rceil
$$

and $y_{n}=0^{k_{n}}$. We then define

$$
f_{\alpha}(X)=x_{1} y_{1} x_{2} y_{2} \cdots x_{n} y_{n} \cdots .
$$


Observe that $f_{\alpha}$ is a continuous function mapping $\mathbf{C}$ to $\mathbf{C}$. We now show that it modifies the dimension of $X$ in a controlled manner.

Lemma 4.12. For any $X \in \mathbf{C}$ and $\alpha \in(0,1)$,

$$
\operatorname{dim}\left(f_{\alpha}(X)\right)=\alpha \cdot \operatorname{dim}(X)
$$

and

$$
\operatorname{Dim}\left(f_{\alpha}(X)\right)=\alpha \cdot \operatorname{Dim}(X) .
$$

Proof. The proof uses (2.5) and (2.6), the Kolmogorov complexity characterizations of dimension and strong dimension.

Let $w \sqsubseteq f_{\alpha}(X)$. For some $n$,

$$
w=x_{1} y_{1} \cdots x_{n-1} y_{n-1} v,
$$

where $v \sqsubseteq x_{n} y_{n}$. Then

$$
\begin{aligned}
K(w) \leq & K\left(x_{1} \cdots x_{n-1}\right)+K(v) \\
& +K\left(k_{1}\right)+\cdots+K\left(k_{n-1}\right)+O(1) \\
\leq & K\left(x_{1} \cdots x_{n-1}\right)+O(n \log n) .
\end{aligned}
$$

Because

$$
|w| \geq\left|x_{1} y_{1} \cdots x_{n-1} y_{n-1}\right| \geq \frac{(n-1)^{2}}{\alpha},
$$

we have

$$
\frac{K(w)}{|w|} \leq \frac{\alpha \cdot K\left(x_{1} \cdots x_{n-1}\right)}{\left|x_{1} \cdots x_{n-1}\right|}+\frac{O(n \log n)}{(n-1)^{2}}
$$

It follows that

$$
\begin{aligned}
\operatorname{dim}\left(f_{\alpha}(X)\right) & \leq \alpha \liminf _{n \rightarrow \infty} \frac{K\left(x_{1} \cdots x_{n-1}\right)}{\left|x_{1} \cdots x_{n-1}\right|} \\
& =\alpha \liminf _{n \rightarrow \infty} \frac{K(x\lceil n)}{n} \\
& =\alpha \cdot \operatorname{dim}(X),
\end{aligned}
$$

where the first equality holds because the block $x_{n}$ is short relative to $x_{1} \cdots x_{n-1}$. Similarly, $\operatorname{Dim}\left(f_{\alpha}(X)\right) \leq \alpha \cdot \operatorname{Dim}(X)$.

For the other inequality, we have

$$
\begin{aligned}
K\left(x_{1} \cdots x_{n-1}\right) \leq & K(w)+K\left(k_{1}\right)+\cdots+K\left(k_{n-1}\right) \\
& +O(1) \\
\leq & K(w)+O(n \log n)
\end{aligned}
$$

and

$$
|w| \leq\left|x_{1} y_{1} \cdots x_{n} y_{n}\right| \leq \frac{n^{2}}{\alpha}+n \leq \frac{(n+1)^{2}}{\alpha},
$$

so

$$
\begin{aligned}
\frac{K(w)}{|w|} & \geq \alpha \frac{K\left(x_{1} \cdots x_{n-1}\right)-O(n \log n)}{(n+1)^{2}} \\
& =\alpha \frac{K\left(x_{1} \cdots x_{n-1}\right)}{\left|x_{1} \cdots x_{n-1}\right|} \frac{(n-1)^{2}}{(n+1)^{2}}-\frac{O(n \log n)}{(n+1)^{2}} .
\end{aligned}
$$


Therefore

$$
\begin{aligned}
\operatorname{dim}\left(f_{\alpha}(X)\right) & \geq \alpha \liminf _{n \rightarrow \infty} \frac{K\left(x_{1} \cdots x_{n-1}\right)}{\left|x_{1} \cdots x_{n-1}\right|} \\
& =\alpha \liminf _{n \rightarrow \infty} \frac{K(x\lceil n)}{n} \\
& =\alpha \cdot \operatorname{dim}(X),
\end{aligned}
$$

and analogously, $\operatorname{Dim}\left(f_{\alpha}(X)\right) \geq \alpha \cdot \operatorname{Dim}(X)$.

The function $f_{\alpha}$ establishes the completeness of $\mathrm{DIM}^{\alpha}$.

Theorem 4.13. For all $\alpha \in(0,1), \mathrm{DIM}^{\alpha}$ is Wadge complete for $\boldsymbol{\Pi}_{3}^{0}$. Therefore it is not a $\boldsymbol{\Sigma}_{3}^{0}$-class, and in particular it is not a $\Sigma_{3}^{0}$-class.

Proof. By Lemma 4.12, $f_{\alpha}$ is a Wadge reduction from DIM ${ }^{1}$ to $\mathrm{DIM}^{\alpha}$. Therefore $\mathrm{DIM}^{\alpha}$ is Wadge complete for $\boldsymbol{\Pi}_{3}^{0}$ by composing $f_{\alpha}$ with the reduction from Theorem 4.10.

As $g_{\alpha}$ is also a Wadge reduction from $\mathrm{DIM}_{\text {str }}^{1}$ to $\mathrm{DIM}_{\mathrm{str}}^{\alpha}$, we have from Theorem 4.6 that $\mathrm{DIM}_{\mathrm{str}}^{\alpha}$ is not a $\boldsymbol{\Sigma}_{2}^{0}$-class for all $\alpha \in(0,1)$. We now prove that $\mathrm{DIM}_{\text {str }}^{\alpha}$ is not even $\boldsymbol{\Sigma}_{3}^{0}$ for all $\alpha \in[0,1)$.

Theorem 4.14. For all $\alpha \in[0,1)$, DIM $\mathrm{str}^{\alpha}$ is Wadge complete for $\mathbf{\Pi}_{3}^{0}$. Therefore $\mathrm{DIM}_{\mathrm{str}}^{\alpha}$ is not a $\Sigma_{3}^{0}$-class, and in particular it is not a $\Sigma_{3}^{0}\left[\Pi_{1}^{0}\right]$-class.

Proof. The proof is similar to that of Theorem 4.10, but uses (2.6), the Kolmogorov complexity characterization of strong dimension. Let $\mathcal{C}=\bigcup_{k} \bigcap_{s} \mathcal{O}_{k, s}$ be a $\boldsymbol{\Sigma}_{3}^{0}$-class and without loss of generality assume that $\mathcal{O}_{k, s} \supseteq \mathcal{O}_{k, s+1}$ for all $k, s$.

Let $\alpha \in(0,1)$. (We will discuss the simpler case $\alpha=0$ later.) We define a continuous function $f: \mathbf{C} \rightarrow \mathbf{C}$ in stages that will Wadge reduce $\mathcal{C}$ to $\overline{\operatorname{DIM}_{\text {str }}^{\alpha}}$. The image $Y=f(X)$ will be the unique sequence extending $Y_{s}$ for all $s$. At stage 0 we define $Y_{0}$ to be the empty sequence.

At stage $s>0$ we consider $X \uparrow s$, and define $k(s)$ as in the proof of Theorem 4.10. There are three substages.

Substage (a). First consider all strings $\rho$ extending $Y_{s-1}$ of minimal length with $K(\rho) \geq \alpha|\rho|$, and take the leftmost one of these $\rho$ 's.

Substage (b). Next consider all strings $\sigma$ extending $\rho$ of minimal length with $K(\sigma) \geq\left(\alpha+\frac{1}{k(s)}\right)|\sigma|$, and take the leftmost one of these $\sigma$ 's. If $k(s)$ does not exist, let $\sigma=\rho$.

Substage (c). Extend $\sigma$ with a block of 0 's to obtain $Y_{s}=\sigma 0^{|\sigma|^{2}-|\sigma|}$.

That is, to define $Y_{s}$, we first select $\rho$ to increase the Kolmogorov complexity rate to $\alpha$. This ensures that $Y$ will have strong dimension at least $\alpha$. We then construct a piece of evidence $\sigma$ that $Y$ has strong dimension at least $\alpha+\frac{1}{k(s)}$. We finish $Y_{s}$ with a long block of 0's to bring the Kolmogorov complexity down to a near-zero rate, so that the next stage will work properly.

If $X \in \mathcal{C}$, then for the minimal $k$ such that $X \in \bigcap_{s} \mathcal{O}_{k, s}$, infinitely many prefixes $\sigma \sqsubseteq Y$ satisfy $K(\sigma) \geq\left(\alpha+\frac{1}{k}\right)|\sigma|$. Therefore $\operatorname{Dim}(Y) \geq \alpha+\frac{1}{k}$, so $Y \notin \operatorname{DIM}_{\text {str }}^{\alpha}$.

Now let $X \notin \mathcal{C}$. Let $\alpha^{\prime}>\alpha$ be arbitrary, and choose $k$ so that $\frac{1}{k}<\alpha^{\prime}-\alpha$. Because $X \notin \mathcal{C}$, we have $k(s)>k$ for all sufficiently large $s$. Let $s_{0}$ be large enough to ensure $k(s)>s$ and $K\left(Y_{s-1}\right) \leq \sqrt{\left|Y_{s-1}\right|}+O(1)<\alpha\left|Y_{s-1}\right|$ hold for all $s \geq s_{0}$. Suppose that

$$
K(w) \geq \alpha^{\prime}|w| \text {. }
$$

holds for some $w$ with $Y_{s-1} \sqsubseteq w \sqsubseteq Y_{s}$ for some stage $s \geq s_{0}$. We then have that $\rho$ is a proper extension of $Y_{s-1}$. By choice of $\rho$ and $\sigma$ and the fact that $\alpha^{\prime}>\alpha+\frac{1}{k}>\alpha+\frac{1}{k(s)}$, we must have $w=\rho$ or $\sigma \sqsubseteq w$. We analyze these two cases separately. 
(i) $w=\rho$ : Let $\rho^{\prime}$ be the string obtained from $\rho$ by removing the last bit. Then $K(\rho) \leq K\left(\rho^{\prime}\right)+$ $O(1)$. By choice of $\rho$, we have $K\left(\rho^{\prime}\right)<\alpha\left|\rho^{\prime}\right|$. We also have $K(\rho) \geq\left(\alpha^{\prime}\right)|\rho|$ by (4.2). Putting these three statements together yields

$$
\alpha^{\prime}|\rho|<\alpha(|\rho|-1)+O(1)
$$

which is a contradiction if $|\rho|=|w|$ is sufficiently large.

(ii) $\sigma \sqsubseteq w$ : Obtain $\sigma^{\prime}$ from $\sigma$ by removing the last bit of $\sigma$. Then we have

$$
\begin{aligned}
K(w) & \leq K\left(\sigma^{\prime}\right)+K(|w|-|\sigma|)+O(1) \\
& \leq K\left(\sigma^{\prime}\right)+\log (|w|-|\sigma|)+O(1) \\
& \leq K\left(\sigma^{\prime}\right)+2 \log |\sigma|+O(1) .
\end{aligned}
$$

By choice of $\sigma, K\left(\sigma^{\prime}\right)<\left(\alpha+\frac{1}{k(s)}\right)\left|\sigma^{\prime}\right|$. These two facts together with (4.2) tell us that

$$
\alpha^{\prime}|w|<\left(\alpha+\frac{1}{k(s)}\right)(|\sigma|-1)+2 \log |\sigma|+O(1),
$$

which is a contradiction for large $|w|$ because $|w| \geq|\sigma|$ and $\alpha^{\prime}>\alpha+\frac{1}{k(s)}$.

Therefore, for all sufficiently long $w \sqsubseteq Y$, (4.2) does not hold. It follows that $\operatorname{Dim}(Y) \leq \alpha$. On the other hand, there are infinitely many $\rho \sqsubseteq Y$ with $K(\rho) \geq \alpha|\rho|$, so $\operatorname{Dim}(Y) \geq \alpha$. Therefore $Y \in \mathrm{DIM}_{\text {str }}^{\alpha}$.

This shows that $f$ is a Wadge reduction from $\mathcal{C}$ to $\overline{\operatorname{DIM}_{\text {str. }}^{\alpha}}$. As $\mathcal{C}$ is an arbitrary $\boldsymbol{\Sigma}_{3}^{0}$-class, this shows that DIM $\mathrm{str}_{\mathrm{s}}^{\alpha}$ is Wadge complete for $\boldsymbol{\Pi}_{3}^{0}$.

The proof for the case $\alpha=0$ is similar, but simpler as substage (a) is omitted in the construction.

\subsection{Ad Hoc Methods}

When classifying classes in the arithmetical hierarchy of reals there are several methods one can use. As we have seen, category methods are sometimes useful up to the third level, Wadge reductions are useful if the classification in the effective (lightface) hierarchy coincides with that in the classical (boldface) hierarchy, and sometimes (as in Proposition 3.1 and Proposition 3.2) one just needs something else. In particular when the level of the class in the effective hierarchy is not the same as the level in the classical hierarchy one often needs to resort to ad hoc arguments. One might think that the notion of effective Wadge reduction, or recursive functional, would be the proper notion to use in classifying classes of reals in the effective hierarchy. However, this notion is rarely useful for the following reason. Let $\mathcal{X}$ be a class without computable elements, such as the class of Martin-Löf random sets or the class of 1-generic sets. Then $\mathcal{X}$ cannot be proven to be complete for any level of the effective hierarchy by a recursive Wadge reduction $f$. For if $X$ is recursive, then so is $f(X)$, so we can never have $X \in \mathbf{C} \Longleftrightarrow f(X) \in \mathcal{X}$. So we see that "easy" classes like $\mathbf{C}$ that contain recursive elements cannot be reduced in such a way to many "difficult" classes, which renders the notion rather useless.

We have left open the question whether $\operatorname{DIM}_{\text {str }}^{1}$ is not in $\Pi_{2}^{0}$, and whether $\operatorname{DIM}_{\text {str }}^{\alpha}$ is not in $\Pi_{3}^{0}$ for any $\Delta_{2}^{0}$-computable $\alpha \in[0,1)$. We have no answer to the second question, but we provide an answer to the first in the next theorem. We make use of the following lemma.

Lemma 4.15. If $\mathcal{X} \in \Pi_{2}^{0}$ is dense then there is a computable $X \in \mathcal{X}$. 
Proof. This is an easy finite extension argument. Suppose that $\mathcal{X}=\left\{X:(\forall m)(\exists k) R^{X}(m, k) \downarrow=\right.$ $1\} \in \Pi_{2}^{0}$ is dense. (Here $R$ is a computable predicate. Note that $R$ does not have to be defined with oracles $X$ that are not in $\mathcal{X}$.) Given any initial segment $\tau$ such that

$$
(\forall n<m)(\exists k) R^{\tau}(m, k) \downarrow=1,
$$

we show how to compute an extension $\sigma \sqsupset \tau$ such that

$$
(\exists k) R^{\sigma}(m, k) \downarrow=1 .
$$

Because $\mathcal{X}$ is dense, there are $X \sqsupset \tau$ and $k$ such that $R^{X}(m, k) \downarrow=1$. Let $u$ be the use of this computation, i.e. the part of the oracle $X$ used in it. Now define $\sigma=\max \{X\lceil u, \tau\}$. Then $\sigma \sqsupseteq \tau$ satisfies (4.3).

Now it is clear that for every $m$ we can compute appropriate extensions $\sigma_{m}$ such that $X=$ $\bigcup_{m} \sigma_{m}$ is computable and $(\forall m)(\exists k) R^{\sigma_{m}}(m, k) \downarrow=1$, so that $X \in \mathcal{X}$.

Theorem 4.16. DIM $\mathrm{D}_{\mathrm{str}}^{1}$ is not a $\Pi_{2}^{0}$-class. Hence it is properly $\Pi_{2}^{0}\left[\Pi_{1}^{0}\right]$.

Proof. Suppose that $\mathrm{DIM}_{\text {str }}^{1}$ is $\Pi_{2}^{0}$. Then, since clearly $\mathrm{DIM}_{\text {str }}^{1}$ is dense, by Lemma 4.15 it contains a computable real, contradicting that every computable real has strong dimension 0 .

We conclude this section by summarizing its main results in the following table.

\begin{tabular}{|c|c|c|}
\hline & $\mathrm{DIM}^{\alpha}$ & $\mathrm{DIM}_{\mathrm{str}}^{\alpha}$ \\
\hline \hline$\alpha=0$ & $\Pi_{2}^{0}-\boldsymbol{\Sigma}_{2}^{0}$ & $\Pi_{3}^{0}\left[\Sigma_{1}^{0}\right]-\boldsymbol{\Sigma}_{3}^{0}$ \\
\hline$\alpha \in(0,1) \cap \Delta_{2}^{0}$ & $\Pi_{3}^{0}-\boldsymbol{\Sigma}_{3}^{0}$ & $\Pi_{3}^{0}\left[\Sigma_{1}^{0}\right]-\boldsymbol{\Sigma}_{3}^{0}$ \\
\hline$\alpha=1$ & $\Pi_{3}^{0}-\boldsymbol{\Sigma}_{3}^{0}$ & $\Pi_{2}^{0}\left[\Pi_{1}^{0}\right]-\left(\boldsymbol{\Sigma}_{2}^{0} \cup \Pi_{2}^{0}\right)$ \\
\hline arbitrary $\alpha \in(0,1)$ & $\mathbf{\Pi}_{3}^{0}-\boldsymbol{\Sigma}_{3}^{0}$ & $\mathbf{\Pi}_{3}^{0}-\boldsymbol{\Sigma}_{3}^{0}$ \\
\hline
\end{tabular}

Question 4.17. Is it the case that $\mathrm{DIM}_{\mathrm{str}}^{\alpha}$ is not in $\Pi_{3}^{0}$ for any $\Delta_{2}^{0}$-computable $\alpha \in[0,1)$ ?

\section{$5 \quad$ Effective Randomness Classes}

We begin this section by pointing out some relationships between computable dimension, Church randomness, and Schnorr randomness.

Analogously to what was done for the constructive case, the computable dimension of a class $\mathcal{A} \subseteq \mathbf{C}$ is defined as

$$
\operatorname{dim}_{\text {comp }}(\mathcal{A})=\inf \left\{\begin{array}{l|l}
s \mid \begin{array}{l}
\text { there exists a computable } \\
s \text {-gale } d \text { for which } \mathcal{A} \subseteq S^{\infty}[d]
\end{array}
\end{array}\right\} .
$$

A selection rule is a function $\varphi:\{0,1\}^{*} \rightarrow\{0,1\}$. With every selection rule $\varphi$ we associate a function $\Phi:\{0,1\}^{*} \rightarrow\{0,1\}^{*}$ defined by $\Phi(\lambda)=\lambda$ and

$$
\Phi(w i)= \begin{cases}\Phi(w) i & \text { if } \varphi(w)=1 \\ \Phi(w) & \text { if } \varphi(w)=0\end{cases}
$$

A set $A$ is called Church random if every substring of $\chi_{A}$ (the characteristic string of $A$ ) defined by a computable selection rule is stochastic, i.e., satisfies the law of large numbers. Consider the following property of selection rules:

$$
\inf _{w \in\{0,1\}^{*}} \frac{|\Phi(w)|}{|w|}>0
$$


A computable null set of exponential order is a set of the form $S^{a^{n}}[d]$, where $d$ is a computable martingale and $a>1$. It is easy to check that a set is not in any computable null set of exponential order if and only if $\{A\}$ has computable dimension 1 . With this observation, we can restate a result of Schnorr as follows.

Theorem 5.1. (Schnorr [22, Satz 17.8]) $\{A\}$ has computable dimension 1 if and only if every substring of $\chi_{A}$ selected by a computable selection rule with the property (5.1) is stochastic.

In particular every Church random sequence is not in any null set of the form $S^{a^{n}}[d]$ where $d$ is computable. In the words of Schnorr [23], "Church random sequences approximate the behavior of Schnorr random sequences."

Proposition 5.2. There are sequences with computable dimension 1 that are not Church random.

Proof. Let $R$ be computably random, and let $D=\left\{2^{n} \mid n \in \mathbb{N}\right\}$ be an exponentially sparse decidable domain. Then $A=R-D$ has computable dimension 1 , but $D$ can be computably selected, so $A$ is not Church random.

We now classify the Schnorr random sequences in the arithmetical hierarchy.

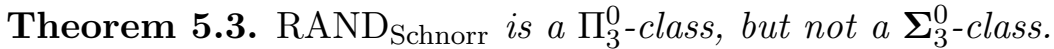

Proof. First note that $\mathrm{RAND}_{\text {Schnorr }} \in \Pi_{3}^{0}: X \in \mathrm{RAND}_{\text {Schnorr }}$ if and only if for every pair of codes $e$ and $f$, either the $e$-th partial computable function $\varphi_{e}$ is not a computable order (i.e. is not total or decreases at some point), $\varphi_{f}$ is not a computable martingale (i.e. is not total or violates the martingale property at some point), or $X \notin S^{\varphi_{e}}\left[\varphi_{f}\right]$, and that every one of these options is $\Sigma_{2}^{0}$.

The rest of the proof resembles that of Theorem 4.10. Fix a (non-computable) sequence of computable martingales $\left\{d_{k}\right\}_{k \in \mathbb{N}}$ and a sequence of computable orders $\left\{h_{k}\right\}_{k \in \mathbb{N}}$ such that

(i) $X \in \mathrm{RAND}_{\text {Schnorr }} \Longleftrightarrow \forall k\left(X \notin S^{h_{k}}\left[d_{k}\right]\right)$.

(ii) $S^{h_{k}}\left[d_{k}\right]-S^{\min \left\{h_{j}: j<k\right\}}\left[\sum_{j<k} d_{j}\right]$ is dense for every $k$.

The $d_{k}$ can be defined by taking appropriate sums of computable martingales so that for any computable martingale $d$, there is some $d_{k}$ such that $d_{k}(w) \geq d(w)$ for all $w$. For the $h_{k}$ one can take any family of computable orders such that every computable order $h$ dominates some $h_{k}$. (Of course the $d_{k}$ and $h_{k}$ cannot be uniformly computable families, but that is of no concern to us.)

Let $\bigcup_{k} \bigcap_{s} \mathcal{O}_{k, s}$ be a $\boldsymbol{\Sigma}_{3}^{0}$-class. We define a continuous function $f: \mathbf{C} \rightarrow \mathbf{C}$ such that

$$
\forall k\left(X \in \bigcap_{s} \mathcal{O}_{k, s} \Longleftrightarrow f(X) \in S^{h_{k}}\left[d_{k}\right]\right)
$$

so that by (i) we have $X \notin \bigcup_{k} \bigcap_{s} \mathcal{O}_{k, s} \Longleftrightarrow f(X) \in \mathrm{RAND}_{\text {Schnorr }}$.

As in the proof of Theorem 4.10 we define the image $Y=f(X)$ in stages. Every time we find a new piece of evidence that $X \in \bigcap_{s} \mathcal{O}_{k, s}$, at stage $s$ say, we build a piece of evidence that $Y \in S^{h_{k}}\left[d_{k}\right]$ by choosing an appropriate finite extension at stage $s$. Such an extension can be found by (ii). The rest of the proof is identical to that of Theorem 4.10.

With only some obvious changes one can also prove the following theorem.

Theorem 5.4. $\mathrm{RAND}_{\mathrm{comp}}$ is a $\Pi_{3}^{0}$-class, but not a $\boldsymbol{\Sigma}_{3}^{0}$-class. 
Proof. Note that $X$ is computably random if and only if for every $e, \varphi_{e}$ is not a computable martingale or $X \notin S^{\infty}\left[\varphi_{e}\right]$, so the class is $\Pi_{3}^{0}$. That it is properly $\Pi_{3}^{0}$ is actually easier than the proof of Theorem 5.3 since we only need the sequence $\left\{d_{k}\right\}$ and not the $\left\{h_{k}\right\}$.

In contrast to the universal constructive supermartingale $\mathbf{d}$ satisfying $\mathrm{RAND}=\overline{S^{\infty}[\mathbf{d}]}$, Theorem

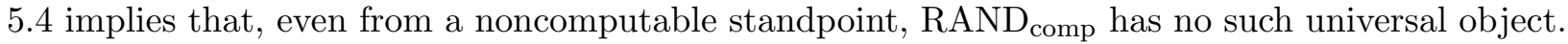
That is, RAND $D_{\text {comp }} \neq \overline{S^{\infty}[d]}$ for any (arbitrarily noncomputable) supermartingale $d$, as otherwise RAND $_{\text {comp }}$ would be a $\Sigma_{2}^{0}$-class.

In this paper we have considered only the extension of the artimetical hierarchy of reals by adding one local quantifier. We end by remarking that one can add of course more local quantifiers. The classes thus obtained also have natural inhabitants. To give an example, again from the theory of randomness, recall that a set $A$ is $n$-random if it is Martin-Löf random relative to $\emptyset^{(n-1)}$. So it is 1-random if it is Martin-Löf random, 2-random if it is Martin-Löf random relative to $K$, etc. Now the class of $n$-random sets is $\boldsymbol{\Sigma}_{2}^{0}$ for every $n$, and in fact one can check that it is $\Sigma_{2}^{0}\left[\Sigma_{n-1}^{0}\right]$.

Acknowledgment. The second and third authors thank Alekos Kechris for a helpful discussion.

\section{References}

[1] K. Ambos-Spies, W. Merkle, J. Reimann, and F. Stephan. Hausdorff dimension in exponential time. In Proceedings of the 16th IEEE Conference on Computational Complexity, pages 210217. IEEE Computer Society, 2001.

[2] K. B. Athreya, J. M. Hitchcock, J. H. Lutz, and E. Mayordomo. Effective strong dimension in algorithmic information and computational complexity. SIAM Journal on Computing. To appear.

[3] J. J. Dai, J. I. Lathrop, J. H. Lutz, and E. Mayordomo. Finite-state dimension. Theoretical Computer Science, 310(1-3):1-33, 2004.

[4] S. A. Fenner. Gales and supergales are equivalent for defining constructive Hausdorff dimension. Technical Report cs.CC/0208044, Computing Research Repository, 2002.

[5] L. Fortnow and J. H. Lutz. Prediction and dimension. Journal of Computer and System Sciences. To appear.

[6] F. Hausdorff. Dimension und äußeres Maß. Mathematische Annalen, 79:157-179, 1919.

[7] J. M. Hitchcock. MAX3SAT is exponentially hard to approximate if NP has positive dimension. Theoretical Computer Science, 289(1):861-869, 2002.

[8] J. M. Hitchcock. Fractal dimension and logarithmic loss unpredictability. Theoretical Computer Science, 304(1-3):431-441, 2003.

[9] J. M. Hitchcock. Gales suffice for constructive dimension. Information Processing Letters, 86(1):9-12, 2003.

[10] C. G. Jockusch. Degrees of generic sets. In Recursion Theory: its Generalizations and Applications, volume 45 of London Mathematical Society Lecture Notes Series, pages 110-139. Cambridge University Press, 1980. 
[11] A. S. Kechris. Classical Descriptive Set Theory. Springer-Verlag, 1994.

[12] H. Ki and T. Linton. Normal numbers and subsets of $\mathbb{N}$ with given densities. Fundamenta Mathematicae, 144:163-179, 1994.

[13] G. Kreisel. Note on arithmetical models for consistent formulae of the predicate calculus. Fundamenta Mathematicae, 37:265-285, 1950.

[14] M. Li and P. M. B. Vitányi. An Introduction to Kolmogorov Complexity and its Applications. Springer-Verlag, Berlin, 1997. Second Edition.

[15] J. H. Lutz. Dimension in complexity classes. SIAM Journal on Computing, 32(5):1236-1259, 2003.

[16] J. H. Lutz. The dimensions of individual strings and sequences. Information and Computation, 187(1):49-79, 2003.

[17] P. Martin-Löf. The definition of random sequences. Information and Control, 9:602-619, 1966.

[18] E. Mayordomo. A Kolmogorov complexity characterization of constructive Hausdorff dimension. Information Processing Letters, 84(1):1-3, 2002.

[19] Y. N. Moschovakis. Descriptive Set Theory. North-Holland, Amsterdam, 1980.

[20] P. Odifreddi. Classical recursion theory, volume 125 of Studies in Logic and the Foundations of Mathematics. North-Holland, 1989.

[21] H. Rogers, Jr. Theory of Recursive Functions and Effective Computability. McGraw - Hill, New York, N.Y., 1967.

[22] C. P. Schnorr. Zufälligkeit und Wahrscheinlichkeit. Lecture Notes in Mathematics, 218, 1971.

[23] C. P. Schnorr. A survey of the theory of random sequences. In R. E. Butts and J. Hintikka, editors, Basic Problems in Methodology and Linguistics, pages 193-211. D. Reidel, 1977.

[24] L. Staiger. Recursive automata on infinite words. In Proceedings of the 10th Annual Symposium on Theoretical Aspects of Computer Science, pages 629-639. Springer-Verlag, 1993.

[25] L. Staiger. On the power of reading the whole infinite input tape. In C. S. Calude and Gh. Paun, editors, Finite Versus Infinite: Contributions to an Eternal Dilemma, pages 335-348. Springer-Verlag, 2000.

[26] L. Staiger. Constructive dimension equals Kolmogorov complexity. Information Processing Letters, 93(3):149-153, 2005.

[27] S. A. Terwijn. Complexity and randomness. Rendiconti del Seminario Matematico di Torino, 62(1):1-38, 2004.

[28] M. van Lambalgen. Random Sequences. PhD thesis, Department of Mathematics, University of Amsterdam, 1987.

[29] J. Ville. Étude Critique de la Notion de Collectif. Gauthier-Villars, Paris, 1939. 
[30] W. W. Wadge. Degrees of complexity of subsets of the Baire space. Notices of the American Mathematical Society, 19:714-715, 1972.

[31] W. W. Wadge. Reducibility and determinateness on the Baire space. PhD thesis, U. C. Berkeley, 1983.

[32] Y. Wang. Randomness and Complexity. PhD thesis, Department of Mathematics, University of Heidelberg, 1996. 